\title{
Dynamic Dark Energy Equation of State (EoS) and Hubble Constant analysis using type Ia supernovae from Union 2.1 dataset.
}

\author{
Syed Faisal ur Rahman ${ }^{1 *}$ \\ ${ }^{1}$ Institute of Space and Planetary Astrophysics (ISPA), \\ University of Karachi $(U o K)$, Karachi, Pakistan \\ Karachi Institute of Technology and Entrepreneurship (KITE),Karachi, Pakistan \\ 2019 \\ October
}

\begin{abstract}
This paper constraints dynamic dark energy equation of state $(\mathrm{EoS})$ parameters using the type Ia supernovae from Union 2.1 dataset. The paper also discusses the dependency of dynamic dark energy EoS parameters on the chosen or assumed value of the Hubble Constant. To understand the correlation between the Hubble Constant values and measured dynamic dark energy EoS parameters, we used recent surveys being done through various techniques such as cosmic microwave background studies, gravitational waves, baryonic acoustic oscillations and standard candles to set values for different Hubble Constant values as fixed parameters with CPL and WCDM models. Then we applied trust region reflective (TRF) and dog leg (dogbox) algorithms to fit dark energy density parameter and dynamic dark energy EoS parameters. We found a significant negative correlation between the fixed Hubble Constant parameter and measured EoS parameter, w0. Then we used two best fit Hubble Constant values (70 and 69.18474) $\mathrm{km} \mathrm{s}^{-1} M p c^{-1}$ based on Chisquare test to test more dark energy EoS parameters like: JBP, BA, PADE-I, PADEII, and LH4 models and compared the results with $\Lambda$-CDM with constant $w_{d e}=-1$, WCDM and CPL models. We conclude that flat $\Lambda$-CDM and WCDM models clearly provide best results while using the BIC criteria as it severely penalizes the use of extra parameters. However, the dependency of EoS parameters on Hubble Constant value and the increasing tension in the measurement of Hubble Constant values using different techniques warrants further investigation into looking for optimal dynamic dark energy EoS models to optimally model the relation between the expansion rate and evolution of dark energy in our universe.
\end{abstract}

*E-mail: faisalrahman36@hotmail.com 


\section{Introduction}

The discovery of the accelerated expansion of the universe [1] [2] [3] revolutionized modern cosmology and answered many questions related to the evolution of our universe. However, we are still trying to understand the ingredient which is likely responsible for the accelerated expansion of the universe i.e. dark energy. Dark energy seems to be something which is not only overcoming the tendency of collapse of the matter in our universe but it is also providing a push for the accelerated expansion of our universe [4]. After the discovery of the accelerated expansion of the universe in the late 1990s by HighZ Supernova and the Supernova Cosmology Project teams [1][2] [3] using the type Ia supernovae, several observations applying various signatures like cosmic microwave background radiation (CMB), baryonic acoustic oscillation (BAO), Cepheid Variables, large scale structures etc. [5] [6][7] [8][9][10], confirmed the accelerated expansion of our universe. Although, these observations confirm that our universe is going through a phase of accelerated expansion but these different observations also presented some serious problems by getting variations in their measurements of the cosmological parameters based on the standard model of cosmology or the $\Lambda$-CDM model [11][12][13] which is providing impetus towards the development of greater interest in non Lambda-CDM model studies [14][15][16]. New standard candles like active galactic nuclei (AGN) and the use of gravitational waves as standard sirens[17][18] are also being explored to get better measurements of cosmological parameters at high redshifts [19].

\section{Cosmology from type Ia Supernova}

Type Ia Supernovae are useful tools to be used as standard candles because of their almost standard absolute magnitude values. Therefore observations of apparent magnitude (m) and redshift (z) for type Ia Supernovae can lead to meausrements of key cosmological parameters: $\Omega \Lambda, \Omega \mathrm{r}$, and $\Omega \mathrm{m}$, the dark energy, radiation and matter density parameters respectively within the Lambda-CDM cosmology framework [4][2] [3][1]. The difference between apparent magnitude $(\mathrm{m})$ and absolute magnitude is known as the distance modulus, [4] [20]:

$$
\mu=m-M
$$

Given a set of assumed cosmological parameters (C), the redshift of an object, its apparent magnitude and luminosity distance DL are linked thus:

$$
m(C, z)=5 \log (D L(C, z))+M+25
$$

Thus luminosity distance and distance modulus are linked:

$$
\mu(C, z)=5 \log [D L(C, z)]+25
$$

Here luminosity distance (DL) is in Mpc.For a spatially flat universe, we can write luminosity distance as:

$$
D L(z)=(1+z) \chi(z)
$$


Where,

$$
\chi(z)=c \eta(z)
$$

is the comoving distance and $\eta(z)$ is conformal loop back time which can be calculated as:

$$
\eta(z)=\int_{0}^{z} \frac{d z^{\prime}}{H\left(z^{\prime}\right)}
$$

Here, $E(z)=\sqrt{\Omega \Lambda I(z)+\Omega r(1+z)^{4}+\Omega m(1+z)^{3}}$ for flat Lambda-CDM model. $\mathrm{I}(\mathrm{z})$ depends on the parametrization of the dark energy equation of state (EoS) and for standard $\Lambda$-CDM model with EoS as $w_{d e}(\mathrm{z})=-1$ (constant), the multiplier I(z) becomes 1 .

We can separate contribution of $\mathrm{H} 0$ and absolute magnitude ' $\mathrm{M}$ ' from the (2) and (3), as [2] [20]:

$$
\overline{\boldsymbol{M}}=M+25+5 \log (c / H 0)+\sigma_{M}
$$

Here 'c' is the speed of light in vacuum and $\sigma_{M}$ is the uncertainty in absolute magnitude of type Ia supernovae. This is often done to marginalize uncertainties arising from measurements of $\mathrm{H} 0$ and $\mathrm{M}$. However, the dominant contributor in these uncertainties is $\mathrm{H} 0$. We are fixing different $\mathrm{H} 0$ s from various surveys to test them for most suitable $\mathrm{H} 0$ for our dataset in relation with the equation of state (EoS) models in discussion which will minimize these uncertainties for the most suitable value of $\mathrm{HO}$. Therefore instead of separating $\overline{\boldsymbol{M}}$, we can fit cosmologies using (2) and (3). The contribution from absolute magnitude uncertainties is very minor if we apply proper fits for coefficients for stretch, color and probability of supernova in data are hosted by galaxies with less than certain threshold mass. We use Union 2.1's compilation [21] magnitude vs redshift table which used fitted values for coefficients of stretch, color and the probability that a particular supernova in dataset was hosted by a low-mass galaxy. The dataset also employs a constant $\mathrm{M} \approx-19.31$ with uncertainties in distance modulus arising from fitting values and systematic contributions mentioned separately as distance modulus error which we incorporated in our model fitting using TRF and dogbox [22] [23] [24] and $\chi^{2}$ analysis, and so it is absorbed in the parameter error bounds provided $\mathrm{H} 0$ is set to an optimal value.

\section{Dataset and Data Analysis Techniques}

For our study, we use Union 2.1 [21] dataset which is publicly shared by Supernova Cosmology Project (SCP)[2] [3]|25]. The dataset is comprised of 580 type Ia supernovae which passed the usability cuts. The dataset is comprised of redshift range $0.015 \leq z \leq 1.414$ with median redshift at $z \approx 0.294$.

We use SciPy's [26] optimize package's trust region reflective (TRF) and dog leg (dogbox) algorithms [22] [23] [24], which are suitable for problems with constraints as in our case, to fit dark energy density parameter and dynamic dark energy EoS parameters for $\Lambda$-CDM, WCDM, CPL,JBP,BA,PADE-I,PADE-II and LH4 models [27][28][29] 
[30][31] [32] [33]. We also apply grid method to obtain log likelihood [34] for WCDM and CPL to compare results obtained through TRF and dog leg methods [22]. We used TRF and dogbox options simultaneously with our selected models and then used the best fit results based on the $\chi^{2}$ values. TRF is an iterative algorithm which based on the first order optimality condition in a bounds constrained non-linear minimization problem which forms a trust region shape based on the direction of the gradient and the distance from the bounds. TRF considers and ellipsoid trst region for bounds. The other variant dogbox (dog-leg) considers a rectangular region for bounds [22] [23] [24]. We applied both TRF and dogbox configurations and selected the optimal set of parameters constraints based on $\chi^{2}$ values for Lambda-CDM,WCDM and CPL methods. Then applied TRF on dynamic dark energy EoS models with more variables as based on our initial test with lesser variable models, TRF is found more robust of the two and almost gives similar results. TRF and dog-leg algorithms are not commonly used in constraining cosmological parameters but we found these methods in general agreement with $\log$ likelihood method which were used for comparison purposes.

\section{Dynamic Dark Energy Equation of State (EoS)}

In order to extend the standard Lambda-CDM model to incorporate dynamic dark energy EoS, we can define $\mathrm{I}(\mathrm{z})$ as [14]|35]:

$$
I(z)=\exp \left(3 \int_{0}^{z} \frac{1+w_{d e}\left(z^{\prime}\right)}{1+z^{\prime}} d z^{\prime}\right)
$$

For the study we tested various dynamic dark energy EoS models.

We started with standard flat Lambda-CDM model with $w_{d e}=-1$ and then tested WCDM model by treating $w_{d e}$ as free parameter. Then we moved towards more complex CPL, JBP, BA,PADE-I,PADE-II and LH4 models [27][28][29] [30][31] [32] [33] with model equations as:

CPL [28] [29]

$$
w_{d e}(z)=w_{0}+w_{a} \frac{z}{(1+z)}
$$

JBP [30][31]

$$
w_{d e}(z)=w_{0}+w_{a} \frac{z}{(1+z)^{2}}
$$

BA [27]

$$
w_{d e}(z)=w_{0}+w_{a} \frac{z(1+z)}{\left(1+z^{2}\right)}
$$

PADE-I [33]

$$
w_{d e}(z)=\frac{w_{0}+w_{a} \frac{z}{(1+z)}}{1 .+w_{b} \frac{z}{(1+z)}}
$$


For $w b=0$, PADE-I reduces to CPL model.

\section{PADE-II [33]}

$$
w_{d e}(z)=\frac{w_{0}+w_{a} \ln \left(\frac{1}{1+z}\right)}{1 .+w_{b} \ln \left(\frac{1}{(1+z)}\right)}
$$

Linder-Huterer (LH4) [32]

$$
w_{d e}(z)=w_{0}+\frac{\left(w_{a}-w_{0}\right)}{1+{\frac{1}{(1+z) a_{t}}}^{1 / T}}
$$

For parameter boundaries for TRF and dog leg analysis, we set $\Omega \Lambda$ boundary between 0.65 and 0.75 . For w0, we set the upper boundary as $w 0<-1 / 3$ which is a pre-condition for accelerated expansion of our universe but for lower limits we first set restrict it to $w 0 \geq-1$ to exclude phantom dark energy [36][37] and keeping it in quintessence regime [4]. Then we set as $\infty<w 0 \leq-1 / 3$ to allow phantom dark energy. This was done to minimize boundary condition bias while running the optimization algorithms. Similarly for wa, we chose two set of boundaries $-5 \leq w a \leq 5$ and $-0.3 \leq w a \leq 0.3$ to avoid localization bias for optimization algorithm. In case of PADE I and II, wb boundaries are set as $-1<w b<0$ while others remain same. In LH4 case, we set both T and $a_{t}$ between and 0 and 1 .

\section{Hubble Constant Value}

The value of Hubble Constant has recently been a topic of great interest in physics and astronomy community. It had been studied in the past like the first precise measurements by Sandage 1958 [38] which gave $\mathrm{H} 0=75$ but recent interest has increased as the measurements of $\mathrm{H} 0$ from cosmic microwave background (CMB), baryon acoustic oscillations (BAO), standard candles and others do not seem to agree with each other [39][12] [7] [40][41][10]. The problem has become even more interesting as the expansion rate is found to be same in all directions by [42] based on 1000 type Ia supernovae samples. Therefore we considered it appropriate to measure CPL and WCDM model parameters by fixing H0 values from Planck 2018, Riess 2018,Abbott et al. 2017,Planck+SNe+BAO-Planck 2018, Planck+BAO/RSD+WL-Planck 2018, HOLiCOW 2018 and DES 2018 [43] [8]. We also fit our own value for Union 2.1 dataset [21] using the kinematic expression from Riess et al. 2016 [44] for luminosity distance with source redshift of $z<0.04$. Figure 1 shows that luminosity distances from (14) is in good agreement with luminosity distances from (4) for $\mathrm{z}<0.04$ using various EoS models.

The kinematic expression from [4] [44] is written as:

$$
D L(z)=\frac{c z}{H 0}\left[1+\frac{\left(1-q_{0}\right) z}{2}-\frac{\left(1-q_{0}-3 q_{0}^{2}+j_{0}\right) z^{2}}{6}+O\left(z^{3}\right)\right]
$$




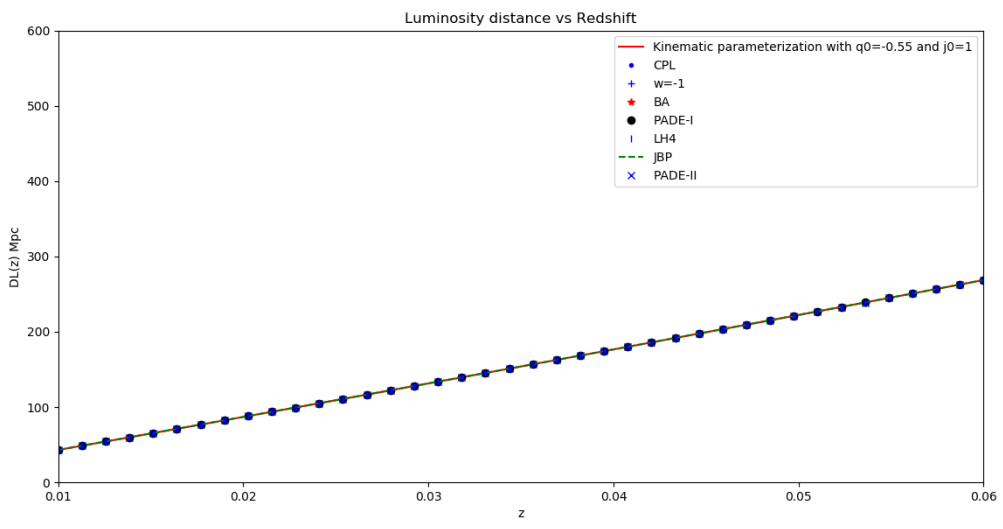

Figure 1: Luminosity distance plots using kinematic expression for DL(z) and comparison with DL(z) using various EoS models and Lambda-CDM with with $w_{d e}(\mathrm{z})$ as a constant value of $\mathrm{w}=-1$.

With $q_{0}=-0.55$ and $j_{0}=1$.

We can see from tables 1 and 2 that our best measurements based on $\chi^{2}$ values for both CPL and WCDM are obtained through $\mathrm{H} 0=70 \mathrm{~km} \mathrm{~s}^{-1} M p c^{-1}$ which is measured by Abbott et al. 2017 by studying gravitational waves (GW170817) [18][17][43] from neutron stars collision and was also measured by Wilkinson Microwave Anisotropy Probe (WMAP) [5] with WMAP only dataset. Our second best measurements were obtained through the best fit $H 0=69.184738270 .50179901$ or approximately 69.185 $\mathrm{km} \mathrm{s}^{-1} M \mathrm{Mc}^{-1}$ value from Union 2.1 dataset using kinematic expression for luminosity distance which is closer to the value obtained by [5] using WMAP+eCMB+BAO+H0 data set [6]. We applied TRF with bounds $65 \leq H 0 \leq 75$ to obtain the best fit $\mathrm{H} 0$ value. Both of these values are interestingly somewhat in the middle region of the $\mathrm{H} 0$ values obtained by early universe studies [45] like Planck cosmic microwave background (CMB) [46] [47] [48][7] or baryon acoustic oscillations (BAO) [49],[9] which give $\mathrm{H} 0 \approx$ 67 and standard candles studies like [1] [50] [44] [51][52] [53][10] which give H0 $>73$. Because of this discrepancy in the measurement of H0,higher redshift studies of type Ia supernovae and other standard candles are becoming important [54][51][55]. Like early universe studies, standard candles are also useful to study the nature of dark energy [56] which is still an open problem of cosmology [57]|34].

In order to understand how $\mathrm{H} 0$ value affects the measurements of dynamic dark energy EoS model parameters, we simply cross-correlated the data in tables 1 and 2 . Figures 2 and 3 show how the measurement or choice of the Hubble Constant can affect the measurements of dynamic dark energy EoS parameters in WCDM and CPL models. We can clearly observe significant negative cross-correlation between w0 and $\mathrm{H} 0$ for both WCDM and CPL models.

These results are particularly interesting due to the Hubble Constant tension arising due to the differences in measurements of $\mathrm{H} 0$ through cosmic microwave background, 
Table 1: Best fit values for WCDM model using union 2.1 dataset

\begin{tabular}{lllll}
\hline WCDM & & & & \\
\hline H0 & $\Omega \Lambda$ & w0 & $\chi^{2}$ & Bounds on $\Omega \Lambda$, w0 \\
\hline 67.400 & 0.75 & -0.7024 & 606.6761 & $(0.65,0.75),(-\infty,-1 / 3)$ \\
67.400 & 0.75 & -0.7024 & 606.6761 & $(0.65,0.75),(-1,-1 / 3)$ \\
73.520 & 0.65 & -1.7459 & 614.5908 & $(0.65,0.75),(-\infty,-1 / 3)$ \\
73.520 & 0.75 & -1.0000 & 740.6163 & $(0.65,0.75),(-1,-1 / 3)$ \\
$\mathbf{7 0 . 0 0 0}$ & $\mathbf{0 . 7 2}$ & $\mathbf{- 1 . 0 0 4 5}$ & $\mathbf{5 6 2 . 2 2 5 7}$ & $(\mathbf{0 . 6 5 , 0 . 7 5}),(-\infty,-\mathbf{- 1 / 3})$ \\
70.000 & 0.72 & -1.0000 & 562.2267 & $(0.65,0.75),(-1,-1 / 3)$ \\
72.500 & 0.65 & -1.5683 & 588.7033 & $(0.65,0.75),(-\infty,-1 / 3)$ \\
72.500 & 0.75 & -1.0000 & 646.1989 & $(0.65,0.75),(-1,-1 / 3)$ \\
67.770 & 0.75 & -0.7353 & 594.2718 & $(0.65,0.75),(-\infty,-1 / 3)$ \\
67.770 & 0.75 & -0.7353 & 594.2718 & $(0.65,0.75),(-1,-1 / 3)$ \\
$\mathbf{6 9 . 1 8 5}$ & $\mathbf{0 . 7 5}$ & $\mathbf{- 0 . 8 6 4 5}$ & $\mathbf{5 6 5 . 9 4 0 2}$ & $(\mathbf{0 . 6 5 , 0 . 7 5}),(-\infty,-1 / 3)$ \\
$\mathbf{6 9 . 1 8 5}$ & $\mathbf{0 . 7 5}$ & $\mathbf{- 0 . 8 6 4 5}$ & $\mathbf{5 6 5 . 9 4 0 2}$ & $(\mathbf{0 . 6 5 , 0 . 7 5}),(-\mathbf{1},-\mathbf{1} / \mathbf{3})$
\end{tabular}

Table 2: Best fit values for CPL model using union 2.1 dataset

\begin{tabular}{|c|c|c|c|c|c|}
\hline \multicolumn{6}{|l|}{ CPL } \\
\hline $\mathrm{HO}$ & $\Omega \Lambda$ & w0 & wa & $\chi^{2}$ & Bounds on $\Omega \Lambda$,w0,wa \\
\hline 66.300 & 0.65 & -0.333 & -3.601 & 620.9512 & $(0.65,0.75),(-\infty,-1 / 3),(-5,5)$ \\
\hline 66.300 & 0.65 & -0.333 & -3.601 & 620.9512 & $(0.65,0.75),(-1,-1 / 3),(-5,5)$ \\
\hline 66.300 & 0.75 & -0.567 & -0.300 & 650.2256 & $(0.65,0.75),(-\infty,-1 / 3),(-0.3,0.3)$ \\
\hline 66.300 & 0.75 & -0.567 & -0.300 & 650.2256 & $(0.65,0.75),(-1,-1 / 3),(-0.3,0.3)$ \\
\hline 67.400 & 0.65 & -0.333 & -4.678 & 585.7344 & $(0.65,0.75),(-\infty,-1 / 3),(-5,5)$ \\
\hline 67.400 & 0.65 & -0.333 & -4.678 & $\mathbf{5 8 5 . 7 3 4 4}$ & $(0.65,0.75),(-1,-1 / 3),(-5,5)$ \\
\hline 67.400 & 0.75 & -0.664 & -0.300 & 602.6112 & $(0.65,0.75),(-\infty,-1 / 3),(-0.3,0.3)$ \\
\hline 67.400 & 0.75 & -0.664 & -0.300 & 602.6112 & $(0.65,0.75),(-1,-1 / 3),(-0.3,0.3)$ \\
\hline 67.770 & 0.65 & -0.419 & -4.326 & 579.2176 & $(0.65,0.75),(-\infty,-1 / 3),(-5,5)$ \\
\hline 67.770 & 0.65 & -0.419 & -4.326 & 579.2176 & $(0.65,0.75),(-1,-1 / 3),(-5,5)$ \\
\hline 67.770 & 0.75 & -0.697 & -0.300 & 590.9692 & $(0.65,0.75),(-\infty,-1 / 3),(-0.3,0.3)$ \\
\hline 67.770 & 0.75 & -0.697 & -0.300 & 590.9692 & $(0.65,0.75),(-1,-1 / 3),(-0.3,0.3)$ \\
\hline 68.340 & 0.65 & -0.584 & -3.491 & $\mathbf{5 7 1 . 3 3 3 3}$ & $(0.65,0.75),(-\infty,-1 / 3),(-5,5)$ \\
\hline 68.340 & 0.65 & -0.584 & -3.491 & $\mathbf{5 7 1 . 3 3 3 3}$ & $(0.65,0.75),(-1,-1 / 3),(-5,5)$ \\
\hline 68.340 & 0.75 & -0.749 & -0.300 & 577.1210 & $(0.65,0.75),(-\infty,-1 / 3),(-0.3,0.3)$ \\
\hline 68.340 & 0.75 & -0.749 & -0.300 & 577.1210 & $(0.65,0.75),(-1,-1 / 3),(-0.3,0.3)$ \\
\hline 69.185 & 0.65 & -0.830 & -2.278 & 564.2394 & $(0.65,0.75),(-\infty,-1 / 3),(-5,5)$ \\
\hline 69.185 & 0.65 & -0.830 & -2.278 & 564.2394 & $(0.65,0.75),(-1,-1 / 3),(-5,5)$ \\
\hline 69.185 & 0.75 & -0.827 & -0.300 & 565.2861 & $(0.65,0.75),(-\infty,-1 / 3),(-0.3,0.3)$ \\
\hline 69.185 & 0.75 & -0.827 & -0.300 & 565.2861 & $(0.65,0.75),(-1,-1 / 3),(-0.3,0.3)$ \\
\hline 70.000 & 0.72 & -1.005 & -0.011 & 562.2257 & $(0.65,0.75),(-\infty,-1 / 3),(-5,5)$ \\
\hline 70.000 & 0.72 & -1.005 & -0.011 & 562.2257 & $(0.65,0.75),(-\infty,-1 / 3),(-0.3,0.3)$ \\
\hline 70.000 & 0.72 & -1.000 & 0.039 & 562.2260 & $(0.65,0.75),(-1,-1 / 3),(-5,5)$ \\
\hline 70.000 & 0.72 & -1.000 & 0.039 & 562.2260 & $(0.65,0.75),(-1,-1 / 3),(-0.3,0.3)$ \\
\hline 72.500 & 0.67 & -1.727 & 2.402 & $\mathbf{5 8 3 . 9 8 1 1}$ & $(0.65,0.75),(-\infty,-1 / 3),(-5,5)$ \\
\hline 72.500 & 0.65 & -1.598 & 0.300 & $\mathbf{5 8 7 . 6 2 1 7}$ & $(0.65,0.75),(-\infty,-1 / 3),(-0.3,0.3)$ \\
\hline 72.500 & 0.75 & -1.000 & -1.142 & 623.7469 & $(0.65,0.75),(-1,-1 / 3),(-5,5)$ \\
\hline 72.500 & 0.75 & -1.000 & -0.300 & 635.4969 & $(0.65,0.75),(-1,-1 / 3),(-0.3,0.3)$ \\
\hline 73.520 & 0.65 & -2.101 & 3.575 & 603.5328 & $(0.65,0.75),(-\infty,-1 / 3),(-5,5)$ \\
\hline 73.520 & 0.65 & -1.773 & 0.300 & 613.0368 & $(0.65,0.75),(-\infty,-1 / 3),(-0.3,0.3)$ \\
\hline 73.520 & 0.75 & -1.000 & -1.950 & 682.8480 & $(0.65,0.75),(-1,-1 / 3),(-5,5)$ \\
\hline 73.520 & 0.75 & -1.000 & -0.300 & 722.6496 & $(0.65,0.75),(-1,-1 / 3),(-0.3,0.3)$ \\
\hline
\end{tabular}




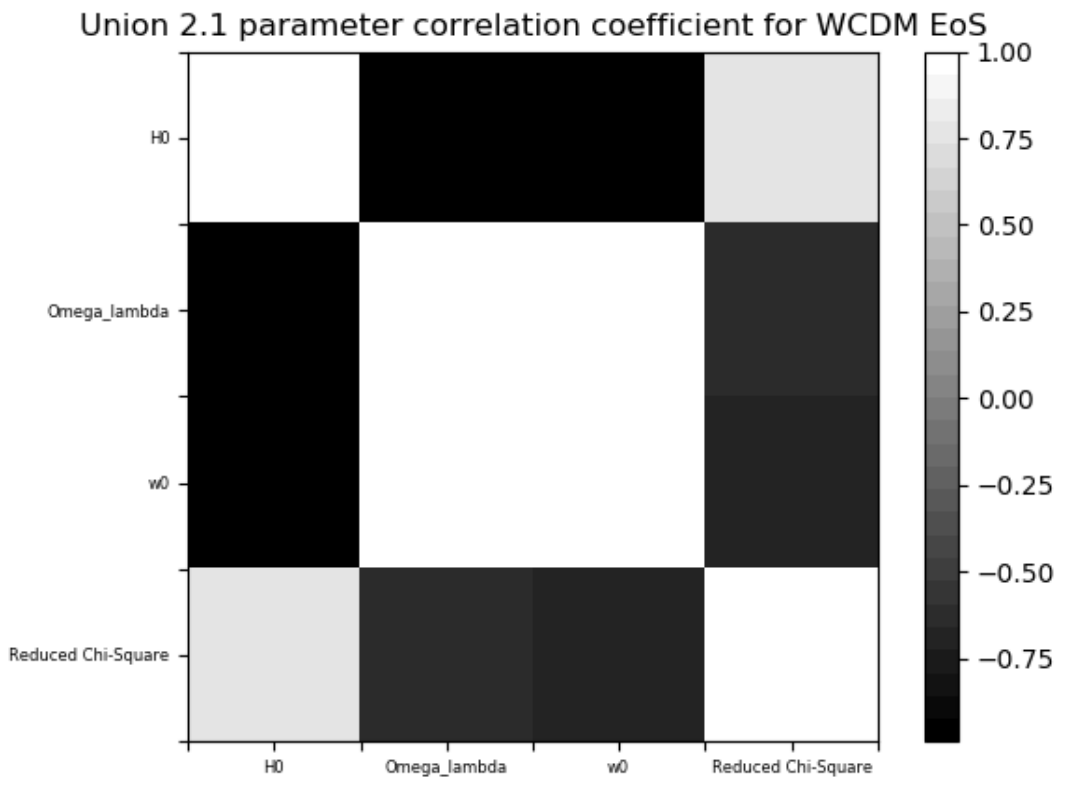

Figure 2: Cross-correlation of WCDM model parameters with H0, $\chi^{2}$ and each other. We can clearly observe significant negative cross-correlation between w0 and $\mathrm{H} 0$. 


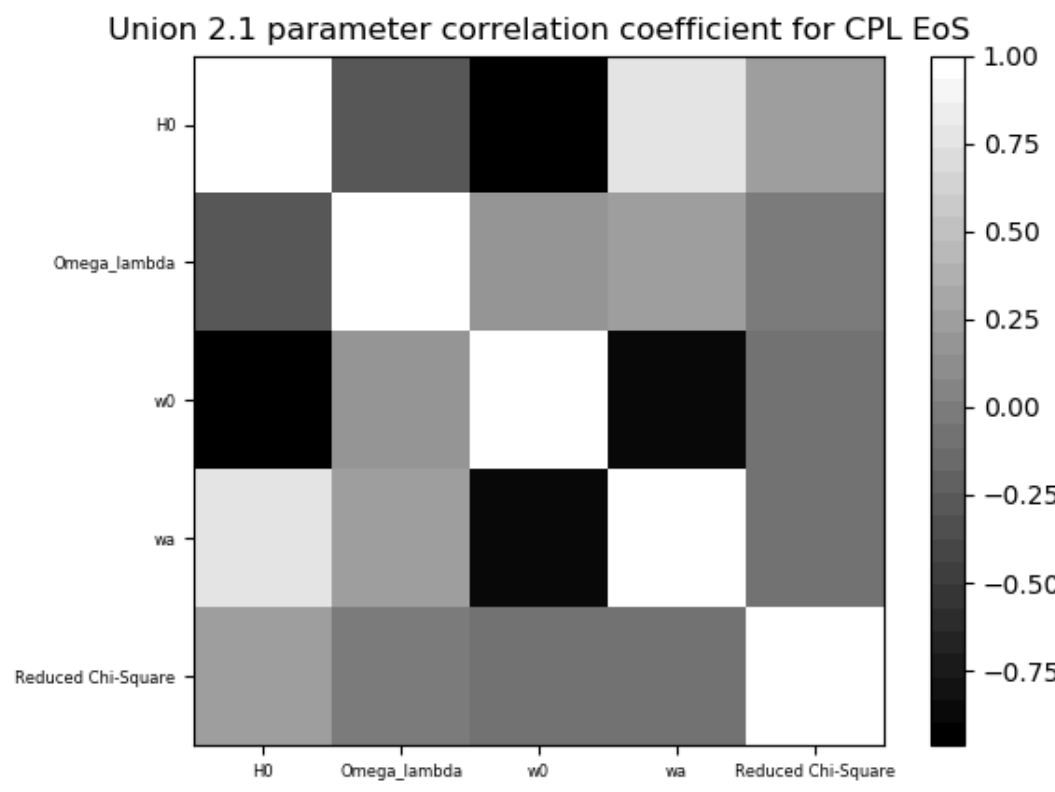

Figure 3: Cross-correlation of CPL model parameters with H0, $\chi^{2}$ and each other. We can again clearly observe significant negative cross-correlation between w0 and H0. We can also observe positive cross-correlation between $\mathrm{HO}$ and wa. 
standard candles and other techniques.

\section{Results}

In figure 4 for WCDM model, the maximum likelihood fit values are $\Omega \Lambda=0.712_{-0.021}^{+0.039}$ and $w 0=-0.995_{-0.073}^{+0.070}$. Their corresponding mean likelihood fit values are $\Omega \Lambda=0.724$ Âś 0.030 and $w 0=-1$ Âś 0.065 . Both maximum and mean likelihood values agree, within one sigma overlapping values, with the best fit values obtained by using TRF and dog leg methods for $\mathrm{H} 0=70$. The best values from tables 1 and 3 are $\Omega \Lambda=0.720362$ Âś 0.0626 and $w 0=-1.00449$ Âś 0.1435 . Values in table 1 are rounded off to fit in the columns.

In figure 5 , for CPL model, the maximum likelihood fit values are $\Omega \Lambda=0.687_{-0.060}^{+0.103}$, $w 0=-0.98_{-0.014}^{+0.014}$ and $w a=-0.35_{-0.92}^{+0.47}$. Their corresponding mean likelihood fit values are $\Omega \Lambda=0.731$ Âś 0.080 , w0=-1.02 Âś 0.015 and wa=0.01 Âś 0.65 . Again both maximum and mean likelihood values agree, within one sigma overlapping values, with the best fit values obtained by using TRF and $\operatorname{dog}$ leg methods for $\mathrm{H} 0=70$. The best values from tables 2 and 3 are $\Omega \Lambda=0.71933$ Âś 0.27885 , w $0=-1.00547$ Âś 0.291303 and wa $=-0.01126$ Âs 3.033239. Values in table 1 are rounded off to fit in the columns. For wa, there is a relatively larger standard deviation in both likelihood estimates and in TRF and dog leg optimization approaches which is likely due to smaller redshift coverage from type Ia supernovae sample from Union 2.1. On very large redshifts, wa almost plays an equal role as w0 in CPL model because on extremely large ' $z$ ' values, $w_{d} e(\mathrm{z})$ approximately becomes $\mathrm{w} 0+$ wa. However in case of a model like JBP, the model will be more or entirely dependent on w0. This means higher redshift surveys especially highly sensitive all sky surveys like galaxy surveys to study the late time integrated Sachs-Wolfe effect (ISW) [58][59][60] or surveys studying the early universe signatures like cosmic microwave background radiation (CMB) or baryonic acoustic oscillations (BAO), can play an important part in estimating parameters like wa or other extended EoS model parameters can make major contributions in higher redshifts in various dynamic dark energy equation of state (EoS) models which are in discussion in this study.

To see how dynamic dark energy EoS evolves in JBP, BA, PADE-I, PADE-II, and LH4 models especially in comparison the results from the flat $\Lambda$-CDM model with constant $w_{d e}=-1$, WCDM and CPL models, we again applied TRF and dog leg methods [22] simultaneously and selected the best fit values based on $\chi^{2}$ criteria.

We can see in figure 6 that for $\mathrm{H} 0=70 \mathrm{~km} \mathrm{~s}^{-1} M p c^{-1}$, the results are closer to $\Lambda$-CDM model with constant $w_{d e}=-1$ except for BA model which is in quintessence regime and PADE-II which is a bit farther than $w_{d e}=-1$ in comparison with others. However, due to large standard deviations from mean for wa, wb, $a_{t}$ and $\mathrm{T}$ parameters in CPL, JBP,BA, PADE-I, PADE-II and LH4 models [27][28][29] [30][31] [32] [33] for relatively smaller redshift objects like in Union 2.1 dataset of type Ia supernovae, we still need to test these models using early universe signatures like CMB and BAO. For our type Ia supernova dataset with relatively smaller redshift coverage in comparison with they early universe studies, we can see that $\Lambda$-CDM model with $w_{d e}=-1$ as fixed value 


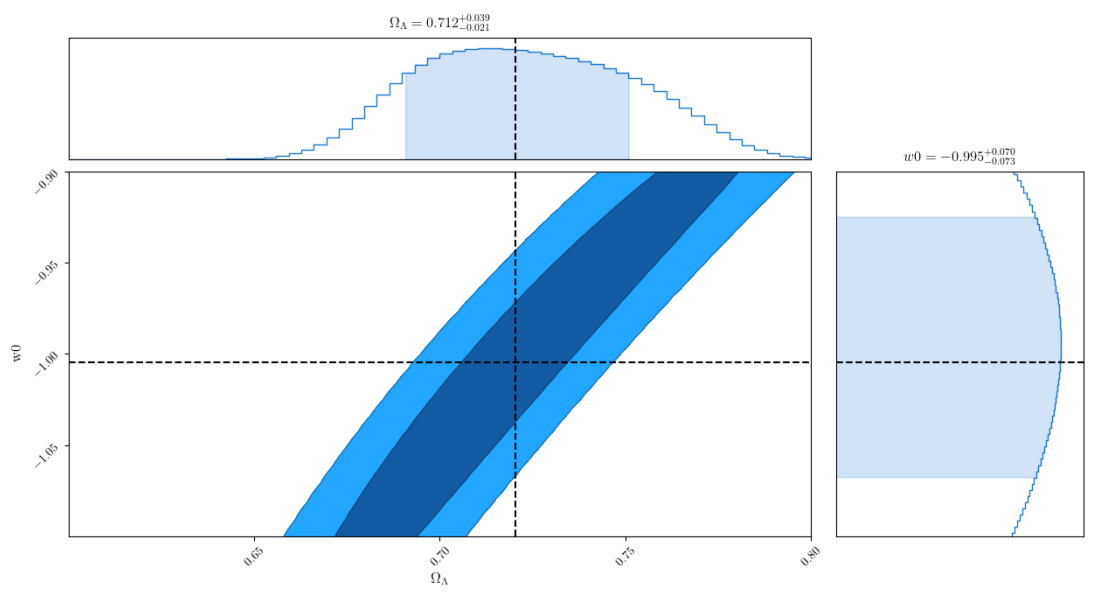

Figure 4: WCDM parameter constraints obtained through maximum likelihood and comparison with results from TRF and dog leg methods (dark dashed lines).

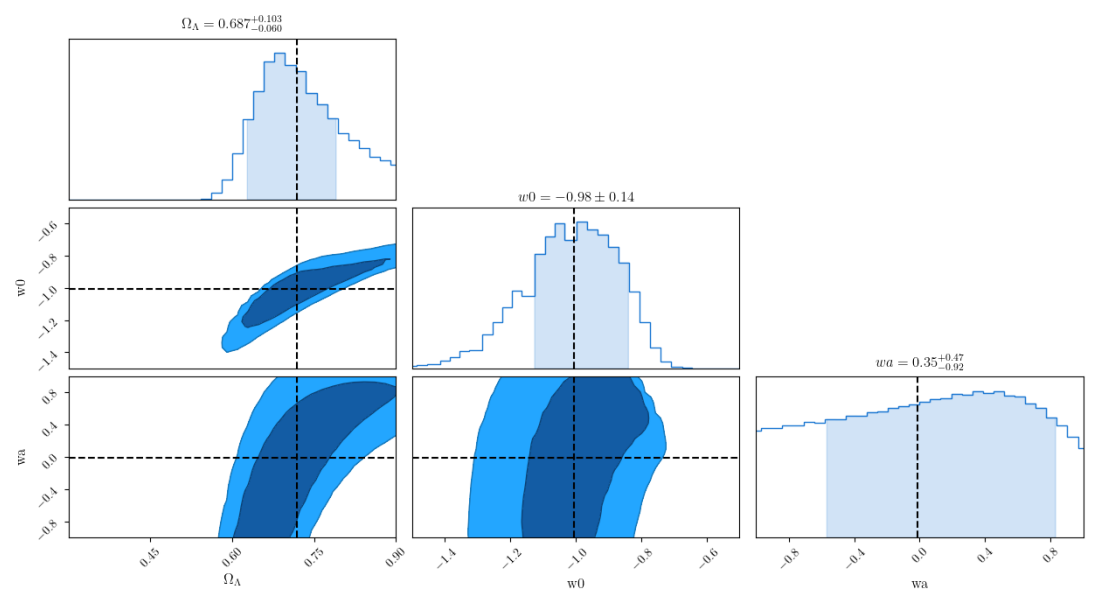

Figure 5: CPL parameter constraints obtained through maximum likelihood and comparison with results from TRF and dog leg methods (dark dashed lines). 


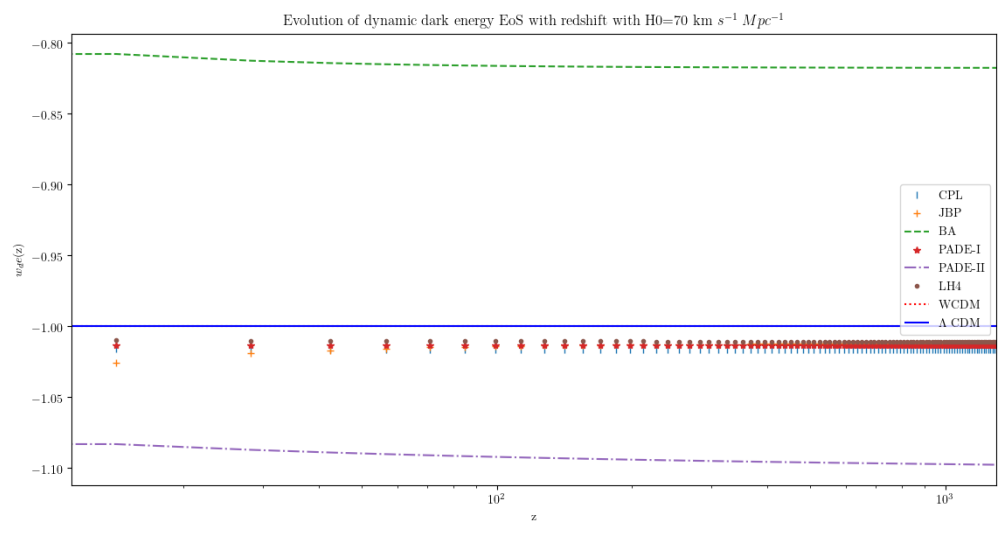

Figure 6: Evolution of $w_{d e}(\mathrm{z})$ for various dynamic dark energy EoS models with redshift for $\mathrm{H} 0=70 \mathrm{~km} \mathrm{~s}^{-1} M p c^{-1}$.

is still the preferred model based on Bayesian information criterion (BIC) [61] [62][63] especially if we consider $\triangle \mathrm{BIC}$ values which are basically the difference of BIC values from our models in discussion with the lowest BIC obtained from these models. $\triangle \mathrm{BIC}$ $>2$ suggests positive evidence against a model with higher BIC and $\Delta \mathrm{BIC}>6$ suggests strong evidence against higher BIC value models [64] as BIC heavily penalizes the inclusion of newer parameters [63] despite having better $\chi^{2}$ scores for non $\Lambda$-CDM models. This can change for higher redshift or early universe studies when extra parameters in dynamic dark energy EoS models are potentially going to play important role which will also be useful for H0 studies [45] [7][10] [54][65][66] .

For $\mathrm{H} 0=69.185 \mathrm{~km} \mathrm{~s}^{-1} M \mathrm{Mc}^{-1}$, we first look at figure 7 and observe that PADE-I is showing most deviation from $w_{d e}=-1$ in comparison with the others especially at higher redshifts. This difference in scale of deviation towards $w_{d e} \ll-1$ is due to the relatively higher contribution of wa and wb of PADE-I model with increasing redshift values. In figure 8 , we remove PADE-I model to see the evolution of $w_{d e}(\mathrm{z})$ in other models. We can see that apart from JBP, which is moving towards quintessence regime, others are closer to phantom regime [36][37] with BA and PADE-II deviating away more from $w_{d e}=-1$ and towards phantom regime. Theoretically,all structures in our universe would be eventually ripped apart by the repulsive forced associated with the phantom dark energy [36][4]. It will be interesting to see if future high precision standard candles, early universe and other surveys can settle expansion rate debate and which $w_{d e}$ evolution or best fit value will be associated with it as we can observe from figures 2 and 3 that expansion rate and dark energy EoS parameters have significant cross-correlation with each other.

We can also see from figures 6,7 and 8 that despite $\mathrm{H} 0$ values being $<2 \%$ different from each other, their impact on $w_{d e}(\mathrm{z})$ evolution is significant for all the models. This difference is significant enough to impact our understanding of the scales and evolution of our universe which warrants the need to carefully model $w_{d e}(\mathrm{z})$ in observations of 


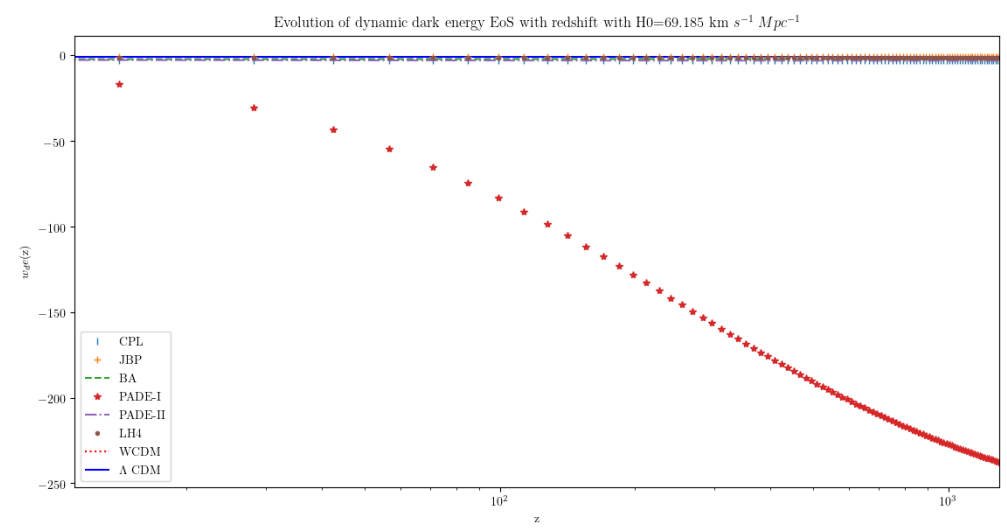

Figure 7: Evolution of $w_{d e}(\mathrm{z})$ for various dynamic dark energy EoS models with redshift for $\mathrm{H} 0=69.185 \mathrm{~km} \mathrm{~s}^{-1} M p c^{-1}$.

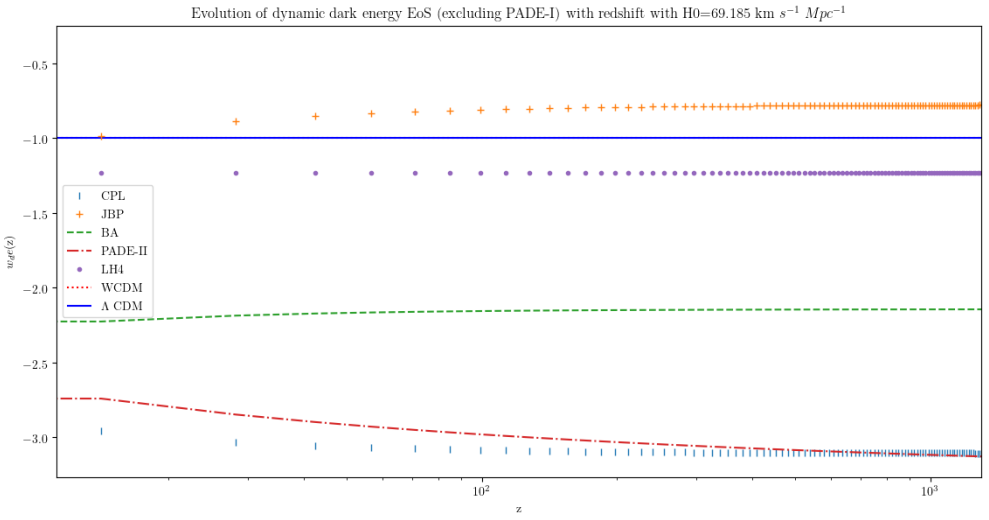

Figure 8: Evolution of $w_{d e}(\mathrm{z})$ for various dynamic dark energy EoS models with redshift for $\mathrm{H} 0=69.185 \mathrm{~km} \mathrm{~s}^{-1} M \mathrm{Mc}^{-1}$ excluding PADE-I. 
early universe signatures, galaxy surveys, standard candles, standard rulers and recently discovered gravitational waves which can be used as standard sirens [67] [68] [13][69]. Gravitational waves can also be used to study the gravitational wave strain signals from type Ia supernovae and we can use them to study cosmological parameters. For this purpose it will be useful to carefully study the progenitors of the type Ia supernovae ([70][13]) as the mass profiles of the objects involved will be crucial in modeling the expected signal [67][13].

\section{Effect of degeneracy between $\mathrm{HO}$ and $\mathrm{M}$ on best fit values}

The difference between results from various $\mathrm{H} 0$ values can also arise from the degeneracy between the Hubble Constant value and uncertainty in absolute value (M) of type Ia supernovae which is shown in equation 6 as $\sigma_{M}$. However if $\mathrm{H} 0$ is properly fit or set then the degeneracy between $\mathrm{H} 0$ and $\mathrm{M}$ should not play much part as $\sigma_{M}$ should be more concerned about the physical properties which relate to the luminosity of the type Ia supernovae in question instead of a cosmological parameter like H0. We can see in figures 9 and 10 is $\sigma_{M} \approx 0$ for $\mathrm{H} 0=70$ especially in mean likelihood case which is less vulnerable to parameter boundary cuts and grid size. However, for $\mathrm{H} \cong 69.185$, the uncertainty increases a bit as shown in figures 11 and 12 . For $\mathrm{H} 0=73.52$ [52] and $\mathrm{H} 0=67.4$ [7], the uncertainty increases further, as shown in figures (13, 14, 15] and 16), which may indicate that for less optimal $\mathrm{H} 0$ values for a given dataset, $\sigma_{M}$ starts working as an offset parameter for the models being tested instead of only representing uncertainties in the absolute value,M. In order to make more effective use of type Ia supernovae as tools to constraint cosmological parameters, we need better approaches to fit magnitude related parameters which are independent of $\mathrm{HO}$ and other cosmological model considerations. However with current available data and techniques, if we apply proper fits for coefficients for stretch, color and probability of supernova in data are hosted by galaxies with less than certain threshold mass then $\sigma_{M}$ should not play much part. For this reason in tables 1/2 and 3 we did not consider $\sigma_{M}$ in our analysis using TRF and dog leg (dogbox) methods. This will prevent $\sigma_{M}$ from working as an offset parameter and will help us in studying the relation between $\mathrm{H} 0$ and dark energy parameters, and so can help in at least testing various $\mathrm{H} 0$ values coming from different studies .

\section{Conclusion}

We studied various dynamic dark energy EoS models and also discussed the key EoS parameter w0 in relation with the Hubble Constant. We also observed strong negative correlation between the Hubble Constant and EoS parameter w0. This relation is also studied in relation with different $\mathrm{H} 0$ values obtained from various surveys adopting different techniques to constraint the cosmological parameters especially $\mathrm{HO}$. We found that the models we tested agreed mostly with standard cosmological model predictions. We also observed that the extended dynamic dark energy equation of state (EoS) models 


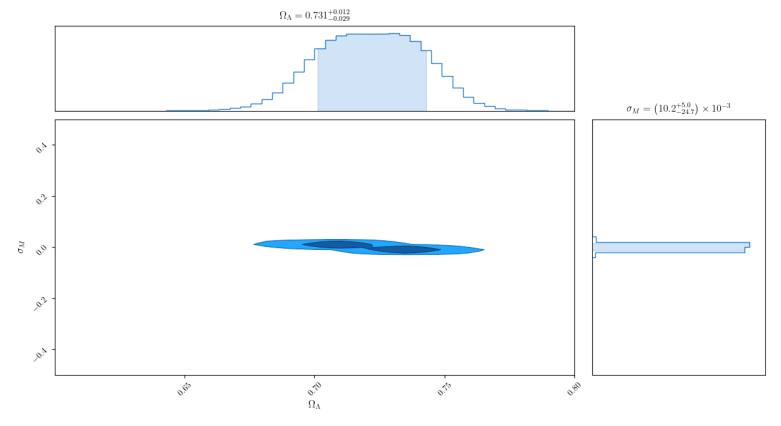

Figure 9: Maximum likelihood estimates for Union 2.1 type Ia supernovae for $\Omega \Lambda$ and $\sigma_{M}$ for $\mathrm{H} 0=70$

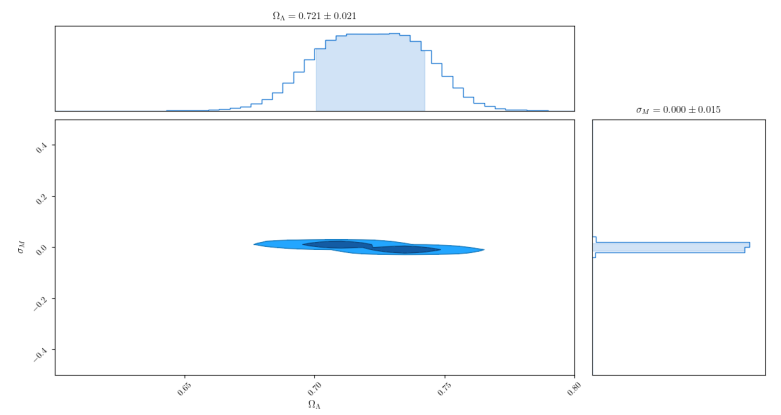

Figure 10: Mean likelihood estimates for Union 2.1 type Ia supernovae for $\Omega \Lambda$ and $\sigma_{M}$ for $\mathrm{H} 0=70$

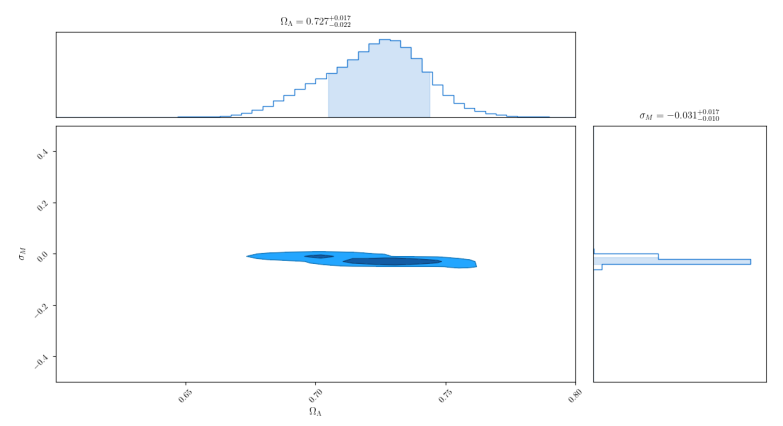

Figure 11: Maximum likelihood estimates for Union 2.1 type Ia supernovae for $\Omega \Lambda$ and $\sigma_{M}$ for $\mathrm{H} 0 \cong 69.185$. 


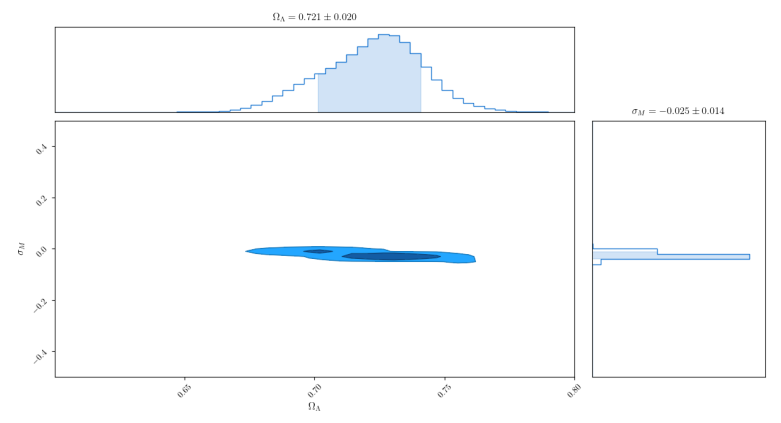

Figure 12: Mean likelihood estimates for Union 2.1 type Ia supernovae for $\Omega \Lambda$ and $\sigma_{M}$ for $\mathrm{H} 0 \cong 69.185$.

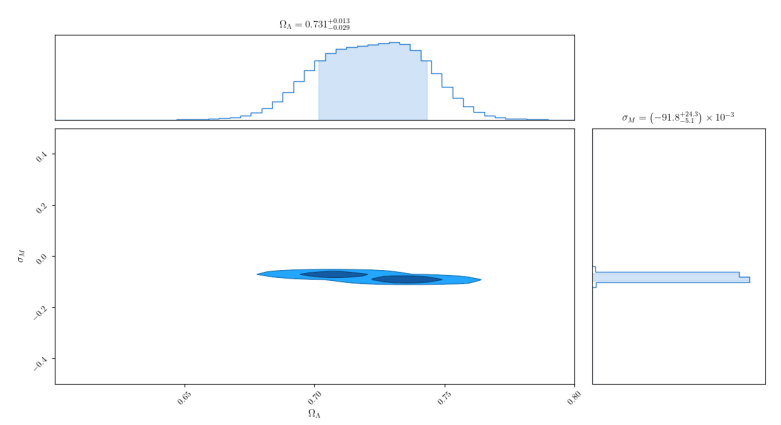

Figure 13: Maximum likelihood estimates for Union 2.1 type Ia supernovae for $\Omega \Lambda$ and $\sigma_{M}$ for $\mathrm{H} 0=67.4$.

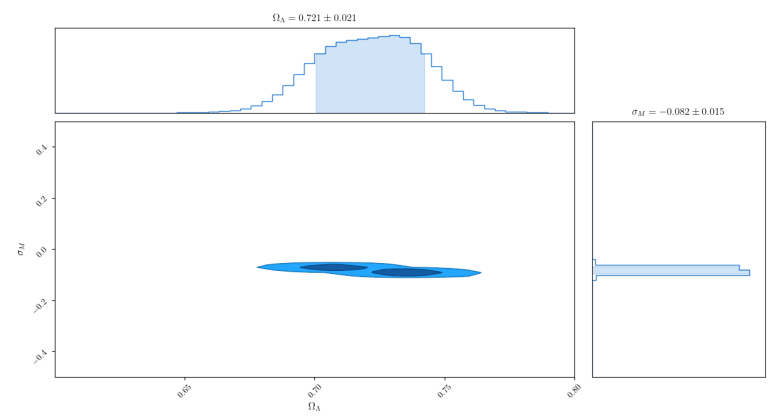

Figure 14: Mean likelihood estimates for Union 2.1 type Ia supernovae for $\Omega \Lambda$ and $\sigma_{M}$ for $\mathrm{H} 0=67.4$. 


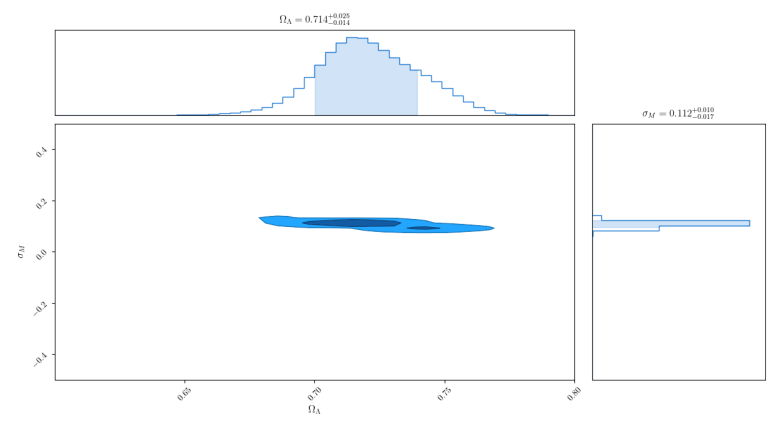

Figure 15: Maximum likelihood estimates for Union 2.1 type Ia supernovae for $\Omega \Lambda$ and $\sigma_{M}$ for $\mathrm{H} 0=73.52$.

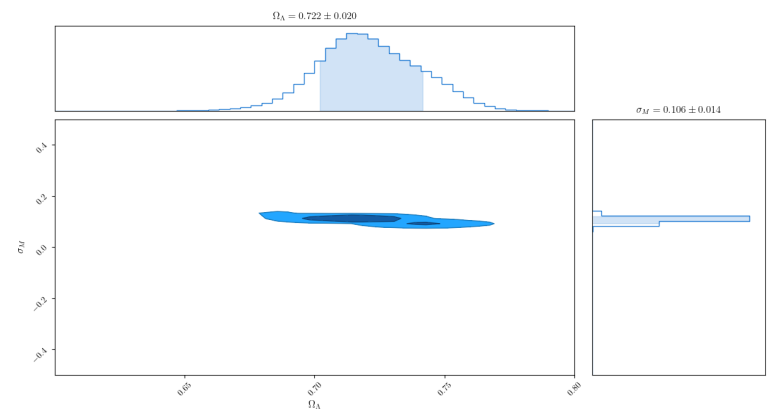

Figure 16: Mean likelihood estimates for Union 2.1 type Ia supernovae for $\Omega \Lambda$ and $\sigma_{M}$ for $\mathrm{H} 0=73.52$. 
we tested agreed with the idea of a universe going through an accelerated expansion phase. We also observed that the value of $\mathrm{w} 0$, which provides value of $w_{d e}(\mathrm{z})$ at $\mathrm{z}=0$ or the current epoch, is in quite close to the standard $\Lambda$-CDM constant value of $w_{d e}=-1$ with $\mathrm{w} 0=-1$ in the confidence interval of one sigma. For the Hubble Constant value of $\mathrm{HO}$ $\approx 69.185$, which we fit on Union 2.1 dataset using kinematic expression for luminosity distance, we found that best fit values for dynamic dark energy EoS models deviate from the constant $w_{d e}=-1$. However, $\Lambda$-CDM with constant value of $w_{d e}=-1$ still comes as the preferable model based on the BIC selection criteria. However this deviation, even in the EoS models with higher number of parameters, shows the importance of studying $\mathrm{H} 0$ in relation with $w_{d e}(\mathrm{z})$. Based on our results, we can also conclude that by carefully

modeling and studying $w_{d e}(\mathrm{z})$, we can potentially resolve the Hubble Constant tension arising from the results obtained using different techniques. However, to achieve this we may need to develop better methods to fit magnitude values independent of $\mathrm{H} 0$ and other cosmological parameters.

\section{Acknowledgement}

I would like to thank Prof. Dr. Jeremy Mould, Emeritus Professor at Swinburne University of Technology for reviewing this work and providing useful suggestions during the development of this paper. 
Table 3: Best Fit Parameters for Dynamic Dark Energy EoS models using Union 2.1 SN type Ia dataset

\begin{tabular}{|c|c|c|c|c|c|c|c|c|c|c|}
\hline $\mathrm{Ho}$ & EoS Models & Omega_Lambda & w0 & $\begin{array}{l}\text { Parameter Values } \\
\text { wa }\end{array}$ & $\mathrm{wb}$ & $a_{t}$ & $\mathrm{~T}$ & Chi-Square & Para & $\mathrm{BIC}$ \\
\hline \multicolumn{11}{|l|}{70} \\
\hline & $\Lambda$-CDM & 0.722287 Áś 0.013 & -1 & & & & & 562.2267 & 1 & 568 \\
\hline & WCDM & $0.720362 \hat{A} \hat{s} 0.0626$ & -1.00449 Âś 0.1435 & & & & & 562.2257 & 2 & 574 \\
\hline & $\mathrm{CPL}$ & 0.71933 Âś 0.27885 & $-1.00547 \hat{\mathrm{A}} \mathbf{s} 0.291303$ & $-0.01126 \hat{A} \leqslant 3.033239$ & & & & 562.2257 & 3 & 581 \\
\hline & $\mathrm{BA}$ & 0.75 Âś 0.86891 & -0.9691 Ấs 1.06464 & 0.15139 Âs 3.56007 & & & & 562.2117 & 3 & \\
\hline & PADE-I & 0.7196 Âs 1.24524 & -1.00523 Âs 1.94515 & $-0.00578414 \hat{A} \hat{s} 4909.4$ & -0.002752 Ấs 4882.5 & & & 562.2257 & 4 & 587 \\
\hline & PADE-II & 0.712876 Âśs 0.4852 & -1.01089 Âs 0.7675 & 1.10857 Âs 271.43 & -0.998715 Âs 267.121 & & & 562.2249 & 4 & 587 \\
\hline & LH4 & $0.72007 \hat{\mathrm{A}} \mathrm{s} 2.0615$ & $-0.999102 \hat{A} \leqslant 2223.47$ & -1.01078 Ấs 314.52 & & 0.9379244 Âśs 29648.86 & 0.960383 Âśs 39515.18 & 562.2258 & 5 & 594 \\
\hline \multicolumn{11}{|c|}{69.185} \\
\hline & $\Lambda$-CDM & 0.6867 Às 0.013664 & -1 & & & & & 567.9516 & 1 & 574 \\
\hline & $\mathrm{CPL}$ & $0.65 \hat{A} \mathbf{s} 0.094414$ & -0.82971 Âs 0.112769 & $-2.27778 \hat{A} \hat{s} 2.70177$ & & & & 564.2394 & 3 & 583 \\
\hline & JBP & 0.65 Âś 0.08967 & 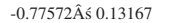 & -3.38651 Âś 3.53197 & & & & 563.8489 & 3 & 582 \\
\hline & BA & 0.65 Âs 0.10221 & -0.88266 Âśs 0.1076 & -1.260334 Âśs 1.7388 & & & & 564.707 & 3 & \\
\hline & PADE-I & 0.66386 Âs 0.12523 & -0.88078055 Ấs 0.11613 & 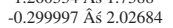 & -0.9958 Âś 0.0000003 & & & 564.1249 & 4 & 89 \\
\hline & PADE-II & 0.65 Âs 0.2274 & -0.81318 Âs 0.5637 & 3.446955 Âś 23.726 & -0.999 Âś 20.04 & & & 564.1126 & 4 & 89 \\
\hline & LH4 & 0.65596 Âs 0.05682 & -0.38368 Âs 0.71809 & -1.23068 Âs 0.28266 & & 0.96887 Âś 0.03171 & 0.000875 Âś 0.26241 & 561.3035 & 5 & \\
\hline
\end{tabular}




\section{References}

[1] A.G. Riess, A. V. Filippenko1, P. Challis, A. Clocchiatti et al., Astron. J., Vol. 116 no. 3 (1998)

[2] S. Perlmutter, G. Aldering, G. Goldhaber, R. A. Knop et al., Astrophys. J. 517 no.2 (1999)

[3] S. Perlmutter ,B. Schmidt, Supernovae and Gamma Ray Bursters, K. Weiler, ed., (Springer-Verlag, New York, 2003)

[4] S. Weinberg, "Cosmology",(Oxford University Press, ISBN 0191523607, 9780191523601, 2008)

[5] C. L. Bennett, D. Larson, J. L. Weiland, N. Jarosik et al., Astrophys. J. Suppl., Volume 208, Issue 2, article id. 20, 54 pp. (2013)

[6] G. Hinshaw D. Larson, E. Komatsu, D. N. Spergel et al., Astrophys. J.Suppl. Ser., 208,19 ( 2013)

[7] Planck Collaboration ,: N. Aghanim, Y. Akrami, M. Ashdown et al., www.cosmos.esa.int. arXiv:1807.06209 (2018)

[8] S. Birrer, T. Treu ,C.E. Rusu , V. Bonvin et al., Monthly Not. Roy. Astron. Soc. 484 (4): $4726 a ̂ A ̆ S ̧ 4753$ (2018)

[9] E. Macaulay, R. C. Nichol, D. Bacon, D. Brout et al. , Monthly Not. Roy. Astron. Soc., Volume 486, Issue 2, Pages 2184âĂŞ2196, https://doi.org/10.1093/mnras/stz978 (2019)

[10] A.G. Riess,C. Stefano, Y. Wenlong, M.M. Lucas ,D. Scolnic, 2019 , arXiv:1903.07603, Astroph. J. accepted (2019)

[11] A. Liddle , "Introduction to modern Cosmology", Second edition (University of Sussex, UK, Wiley Publication, 2003)

[12] N. Jackson, Living Rev Relativ 18: 2. https://doi.org/10.1007//rr-2015-2 (2015)

[13] Rahman S.F. Astron. \& Geophy., Volume 59, Issue 2, Pages 2.39âĂŞ2.42 (2018)

[14] A. Zhai,M. Blanton ,A. Slosar ,J. Tinker, Astrophys. J., Volume 850, Issue 2, article id. 183, 32 pp. (2017)

[15] N. Khosravi, S. Baghram ,N. Afshordi ,N. Altamirano „Phys. Rev. D, Volume 99, Issue 10, id.103526 (2019)

[16] P.J. SolÃăă, A. GÃşmez-Valent, J. de Cruz PÃlrez, Phys. Dark Universe, Volume 25, article id. 100311 (2019)

[17] B.P. Abbott et al., R. Abbott, T. D. Abbott, M. R. Abernathy et al., Phys.Rev.Lett. 116 no.6, 061102 arXiv:1602.03837 [gr-qc] LIGO-P150914 (2016) 
[18] B. P. Abbott, R. Abbott, T. D. Abbott, F. Acernese et al., Nature. adv. online pub. (7678): 85âĂŞ88. arXiv:1710.05835 (2017)

[19] D. Watson ,K.D. Denney, M. Vestergaard, T. M. Davis, Astrophys. J. 740, L49 (2011)

[20] T.M. Davis , Practical Statistics for Astrophysicists,Harley Wood Winter School $2012(2012)$

[21] N. Suzuki, D. Rubin, C. Lidman, G. Aldering et al.,The Supernova Cosmology Project, Astrophys. J., Volume 746, Issue 1, article id. 85, 24 pp.(2012)

[22] C. Voglis , I.E.Lagaris, WSEAS International Conference on Applied Mathematics, Corfu, Greece (2004)

[23] M. A. Branch, T. F. Coleman, Y. Li, SIAM Journal on Scientific Computing, Vol. 21, Number 1, pp 1-23, https://doi.org/10.1137/S1064827595289108 (1999)

[24] J. Nocedal, S. J. Wright, âĂIJNumerical optimization, 2nd editionâĂİ, Springer Series in Operations Research and Financial Engineering, (Springer, New York,2006)

[25] R. Amanullah, C. Lidman, D. Rubin, G. Aldering et al., Astrophys.J.716:712-738 (2010)

[26] E. Jones, E. Oliphant , P. Peterson, http://www.scipy.org/ (2001)

[27] E. M. Barboza Jr. , J.S. Alcaniz , Phys. Rev. B 666415 (2008)

[28] M. Chevallier, D. Polarski,Int. J. Mod. Phys. D 10, 213, [gr-qc/0009008] (2001)

[29] E.V. Linder , Phys. Rev. Lett., 90, 091301, astro-ph/0311403] (2003)

[30] H.K. Jassal , J.S. Bagla , T. Padmanabhan , Monthly Not. Roy. Astron. Soc. 356 L11 (2005)

[31] H.K. Jassal , J.S. Bagla , T. Padmanabhan, Phys. Rev. D 72103503 (2005)

[32] E. V. Linder, D. Huterer, Phys. Rev. D 72, 043509 (2005)

[33] H. Wei, X.P. Yan, Y.N. Zhou , J. Cosm. Astropart. Phys. 1401, 045, 1312.1117 (2014)

[34] T.M. Davis, D. Parkinson, Characterizing Dark Energy Through Supernovae. In: Alsabti A., Murdin P. (eds) Handbook of Supernovae. (Springer, doi:10.1007/9783-319-20794-0_106-1,New York, 2016)

[35] W.D Heacox ., The Expanding Universe: A Primer on Relativistic Cosmology, (Cambridge University Press,UK,2015)

[36] A. Vikman, Phys. Rev. D. 71 (2): 023515 (2005)

[37] J. S. Farnes , Astron. and Astrophys. 620: A92 (2018) 
[38] A. R. Sandage , Astrophys. J., vol. 127, p.513 (1958)

[39] W. L. Freedman, Nature Astron., Volume 1, id. 0121 (2017)

[40] R. Wojtak, A. Agnello, Monthly Not. Roy. Astron. Soc., Volume 486, Issue 4, p.5046-5051 ( 2019)

[41] K. Vattis ,S. M. Koushiappas , L. Abraham, Phys. Rev. D, Volume 99, Issue 12, id.121302 (2019)

[42] J. Soltis , A. Farahi , D. Huterer ,C.M. Liberato ,Phys. Rev. Lett. 122, 091301 (2019)

[43] B. P. Abott, R. Abbott, T. D. Abbott, F. Acernese et al. , Phys.Rev.Lett. 119, 161101 (2017)

[44] A. G. Riess , L. M. Macri , S. L. Hoffmann, D. Scolnic et al., Astrophys. J., 826, 56 (2016)

[45] Gorbunov S., Rubakov V.A. , âĂIJIntroduction to the Theory of the Early Universe: Cosmological Perturbations and Inflationary TheoryâĂİ, (World Scientific, Singapore 2011)

[46] Planck Collaboration, P. A. R. Ade, N. Aghanim, M. I. R. Alves et al. , Astron. and Astroph. 571: A1 (2014)

[47] Planck Collaboration, P. A. R. Ade, N. Aghanim, C. Armitage-Caplan, et al., Astron. and Astroph., Volume 571, id.A23, 48 pp. ( 2014)

[48] Planck Collaboration, R. Adam, P. A. R. Ade, N. Aghanim et al., Astron. and Astroph. 594, A1 ( 2016)

[49] J. N. Grieb , S. Ariel , S. Salazar-Albornozr,Monthly Not. Roy. Astron. Soc., Volume 467, Issue 2, p.2085-2112 (2017)

[50] A.G. Riess, L. Strolger, S. Casertano, H.C. Ferguson et al., Astrophys.J. 659 98-121 astro-ph/0611572 46455850950 (2007)

[51] A.G Riess, S. A. Rodney, D.M. Scolnic, D. L. Shafer et al., Astrophys. J., Volume 853 , Issue 2, article id. 126, 15 pp. (2018)

[52] A.G. Riess , S. Casertano, Y. Wenlong, L. Macri et al., Astrophys. J. 861 (2): 126 (2018)

[53] G. Pietrzy ̊̊̌́ski , D. Graczyk , A. Gallenne, W. Gieren et al. , Nature, 567, 200. https://doi.org/10.1038/s41586-019-0999-4 (2019)

[54] G. Risaliti , E. Lusso, Nature Astron. volume 3, pages272-277 (2019)

[55] D. Scolnic, S. Perlmutter, G. Aldering, D. Brout et al., Astro2020: Decadal Survey on Astronomy and Astrophysics, science white papers, no. 270, arXiv:1903.05128 (2019) 
[56] W.M. Wood-Vasey, G. Miknaitis, C. W. Stubbs, S. Jha et al., Astrophys.J.666:694715, arXiv: astro-ph/0701041(2007)

[57] T.M. Davis, E. MÃúrtsell, J. Sollerman, A. C. Becker et al.,Astrophys. J., Volume 666, Issue 2, pp. 716-725., astro-ph/0701510 (2007)

[58] R.K. Sachs ,A.M. Wolfe, Astrophys. J., 147, 73 (1967)

[59] N. Afshordi, Phys. Rev. D,70,083536 (2004)

[60] S.F. Rahman,M.J. Iqbal,Eur. Phys. J. Plus 134: 302. https://doi.org/10.1140/epjp/i2019-12669-y (2019)

[61] G.E. Schwarz , Annals of Statistics, 6 (2): 461âĂŞ464, doi:10.1214/aos/1176344136 (1978)

[62] F. Arevalo,A. Cid ,J. Moya , Eur. Phys. J. C 77: 565. https://doi.org/10.1140/epjc/s10052-017-5128-7 (2017)

[63] A.R. Liddle, Monthly Not. Roy. Astron. Soc. Lett., Volume 377, Issue 1, pp. L74-L78 (2007)

[64] R. E. Kass, A.E. Raftery, Journal of the American Statistical Association, 90 (430): 773âĂŞ795, doi:10.2307/2291091, ISSN 0162-1459, JSTOR 2291091 (1995)

[65] V. Poulin, T.L. Smith, T. Karwal, M. Kamionkowski, Phys. Rev. Lett., Volume 122, Issue 22, id.221301 (2019)

[66] B. Liu, Z. Li, Z. Zhu, Monthly Not. Roy. Astron. Soc., Volume 487, Issue 2, Pages 1980âĂŞ1985, https://doi.org/10.1093/mnras/stz1179 (2019)

[67] B. F. Schutz Class. Quantum Grav. 16 A131, Number 12A (1999)

[68] M. Jarvis , D. Bacon, C. Blake, M.L. Brown et al., SKA Cosmology Chapter, Advancing Astrophysics with the SKA (AASKA14) Conference, Giardini Naxos (Italy), June 9th-13th 2014', arXiv:1501.03825 (2014)

[69] H. Chen,M. Fishbach,D.E. Holz, Nature, Volume 562, Issue 7728, p.545-547 (2018)

[70] M. Keiichi,T. Yukikatsu,Int.Journ. Mod.n Phys. D, Vol. 25, No. 101630024 (2016) 